\title{
Image-Based Robust Control of Robot Manipulators with Integral Actions
}

\author{
Min Seok Jie and Kang Woong Lee \\ School of Electronics, Telecommunication and Computer Engineering, \\ Hankuk Aviation University, 200-1, Hwajeon-dong, Deokyang-gu, \\ Koyang-city, Kyonggi-do, 412-791, Korea \\ Tel.: +82 23158 0166; Fax: +82 231599257 \\ tomsey@korea.com, kwlee@mail.hangkong.ac.kr
}

\begin{abstract}
In this paper, we propose a robust visual feedback controller with integral action for tracking control of n-link robot manipulators in the presence of constant bounded parametric uncertainties. The proposed control input has robustness to the parametric uncertainty and reduces tracking error in the steady-state. The stability of the closed-loop system is shown by Lyapunov method. The effectiveness of the proposed method is shown by simulation and experiment results on the 5-link robot manipulators with two degree of freedom.
\end{abstract}

Keywords: robust control, visual feedback, integral action, robot manipulator.

\section{Introduction}

Applications of visual based robot control have been increased when the robot is working in unstructured environments. The use of visual feedback in these applications is an attractive solution for the position and motion control of robot manipulators. A visual servo control scheme can be classified in two configurations: fixed-camera, where the visual servoing camera is fixed with respect to the world coordinate frame and camera-in-hand, where the camera is mounted on the endeffector of the robot manipulator [1], [2]. In the camera-in-hand configuration, the camera supplies visual information of the object in the environment to the controller. The objective of this visual feedback control scheme is to move the robot manipulator in such a way that the projection of an object be at a desired position in the image plane obtained by the camera.

The manipulator vision system whose dynamics do not interact with the visual feedback loop can not achieve high performance for high speed tasks. In order to overcome this drawback, the controller must take into account the dynamics of robot manipulators [3]. The robot dynamics, however, includes parametric uncertainties due to load variations and disturbances. A visual servo controller taking into account robot dynamics must be robust to the parametric uncertainties and disturbances. Kelly [4] proposed an image-based direct visual servo controller for camera-in-hand robot manipulators, which is of a simple structure based on a transpose Jacobian term plus gravity compensation. In [5], a robust tracking controller has been designed to 
compensate the uncertainty in the camera orientation and to ensure globally uniformly ultimate boundedness.

Robust control schemes require high feedback gains in order to reduce the tracking error. In practice, high feedback gains are limited because of hardware issues such as digital implementation, actuator saturation and noise contained in velocity measurements. Limitation of feedback gains induces large tracking errors. This problem can be overcome by integral control [6].

In this paper, we design a robust visual servo controller with integral action to compensate parametric uncertainties due to load variations or disturbances and to reduce tracking error. The closed-loop stability including the whole robot dynamics is shown by the Lyapunov method. The proposed robust visual servo controller is applied on a two degree of freedom 5-link robot manipulator to show the performance of the closed-loop system. The simulation and experimental results show convergence behavior of the image feature points.

This paper hereafter is organized as follows. In Sections 2 present the robot and camera models. The proposed robust control system with integral action is analyzed in Section 3. In Section 4 we introduce simulation and experiment results on a two degrees of freedom robot manipulators. The paper is finally summarized in Section 5.

\section{Robot Model and Camera Model}

In this work, we consider a robot manipulator with end effector which a camera is mo unted on. The mathematical model of this system consists of the rigid robot dynamic model and the camera model.

In the absence of friction and disturbance, the dynamic equation of an $n$-link rigid $r$ obot manipulator can be expressed as [7]

$$
M(q) \ddot{q}+C(q, \dot{q}) \dot{q}+G(q)=\tau
$$

where $q \in R^{n}$ is the vector of joint displacements, $\tau \in R^{n}$ is the vector of torques applied to the joints, $M(q) \in R^{n \times n}$ is the symmetric positive definite inertia matrix, $C(q, \dot{q}) \dot{q} \in R^{n}$ is the vector of centripetal and Coriolis torques, and $G(q) \in R^{n}$ is the vector of the gravitational torques. The robot dynamic model (1) has the following properties.

Property 1: For the unknown constant parameter vector $\theta \in R^{p}$, the dynamic equation (1) can be expressed linearly

$$
M(q) \ddot{q}+C(q, \dot{q}) \dot{q}+G(q)=Y(q, \dot{q}, \ddot{q}) \theta=\tau
$$

where $Y(q, \dot{q}, \ddot{q}) \in R^{n \times p}$ is the known regression matrix.

The dynamic equation (1) can be changed to the error dynamic equation. Defining the tracking error as $\tilde{q}=q-q_{d}$, the error dynamic equation for the robot manipulator of (1) is given by 


$$
\ddot{\tilde{q}}=M^{-1}(q)\left[-M(q) \ddot{q}_{d}-C(q, \dot{q}) \dot{q}-G(q)+\tau\right]
$$

where $q_{d}$ is the twice continuously differentiable desired trajectory.

In order to include integral action in the controller, let us define the new state vector

$$
\sigma=\int_{0}^{t} \tilde{q}(\tau) d \tau
$$

where $\sigma=\left[\sigma_{1} \sigma_{2} \cdots \sigma_{n}\right]^{T}$.

Defining the augmented state vector as $\zeta=\left[\begin{array}{lll}\sigma^{T} & \tilde{q}^{T} & \dot{\tilde{q}}^{T}\end{array}\right]^{T}$, the augmented state equation is given by

$$
\dot{\zeta}=A \zeta+B M^{-1}(q)\left[-Y\left(q, \dot{q}, \ddot{q}_{d}\right) \theta+\tau\right]
$$

where

$$
A=\left[\begin{array}{ccc}
0 & I_{n \times n} & 0 \\
0 & 0 & I_{n \times n} \\
0 & 0 & 0_{n \times n}
\end{array}\right], B=\left[\begin{array}{c}
0 \\
0_{n \times n} \\
I_{n \times n}
\end{array}\right] \text {, and } Y\left(q, \dot{q}, \ddot{q}_{d}\right) \theta=M(q) \ddot{q}_{d}+C(q, \dot{q}) \dot{q}+G(q)
$$

The motion dynamics of the image feature point described by the robot joint velocity as

$$
\begin{gathered}
\dot{\xi}=J(q, \xi, Z) \dot{q} \\
\text { where } J(q, \xi, Z)=J_{\text {img }}(q, \xi, Z)\left[\begin{array}{cc}
{ }^{c} R_{w} & 0 \\
0 & { }^{c} R_{w}
\end{array}\right]\left[\begin{array}{cc}
I & 0 \\
0 & T(q)
\end{array}\right] J_{A}(q)
\end{gathered}
$$

In robot control with visual feedback, the control problem is to design a controller to move the end-effector in such a way that the actual image features reach the desired ones specified in the image plane. Let us denote with $\xi_{d}$ the desired image feature vector which is assumed to be constant. We define the image feature error as

$$
\tilde{\xi}=\xi-\xi_{d}
$$

Using (7), the time derivative of (9) can be expressed by

$$
\dot{\tilde{\xi}}=J(q, \xi, Z) \dot{q}=J(q, \xi, Z)\left(\dot{\tilde{q}}+\dot{q}_{d}\right)
$$

We take the desired joint velocity $\dot{q}_{d}$ as

$$
\dot{q}_{d}=-J^{+}\left(q, \xi_{d}, Z\right) K_{c} \xi
$$

where $K_{c}$ is the positive definite matrix and $J^{+}\left(q, \xi_{d}, Z\right)$ is the pseudo inverse matrix defined by $J^{+}\left(q, \xi_{d}, Z\right)=\left[J^{T}\left(q, \xi_{d}, Z\right) J\left(q, \xi_{d}, Z\right)\right]^{-1} J^{T}\left(q, \xi_{d}, Z\right)$.

Substituting (11) into (10) leads to

$$
\dot{\tilde{\xi}}=J(q, \xi, Z) \dot{\tilde{q}}-J(q, \xi, Z) J^{+}\left(q, \xi_{d}, Z\right) K_{c} \xi
$$




\section{Robust Control with Visual Feedback}

In this section, we consider a robust visual feedback controller including integral action in order to compensate the bounded constant parametric uncertainties of robot manipulators. The proposed controller is given by

$$
\tau=Y\left(q, \dot{q}, \ddot{q}_{d}\right) \theta_{o}-M_{o}(q) K \zeta+J^{+}\left(q, \xi_{d}, Z\right) K_{i} \tilde{\xi}+\tau_{N}
$$

where $K$ and $K_{i}$ is the symmetric positive definite gain matrices, $\tau_{N}$ is an additional nonlinear control input compensating for the bounded parametric uncertainties, $M_{o}(q)$ is the nominal matrix of $M(q), \theta_{o}$ is the nominal value of the unknown parameter vector $\theta$ and $Y\left(q, \dot{q}, \ddot{q}_{d}\right) \theta_{o}$ is defined as

$$
Y\left(q, \dot{q}, \ddot{q}_{d}\right) \theta_{o}=M_{o}(q) \ddot{q}_{d}+C_{o}(q, \dot{q}) \dot{q}+G_{o}(q)
$$

where $C_{o}(\cdot)$ and $G_{o}(\cdot)$ are the nominal matrices of $C(\cdot)$ and $G(\cdot)$, respectively.

Substituting the proposed input torque (13) into the equation (5) leads to

$$
\begin{aligned}
\dot{\zeta}=(A-B K) \zeta+B M^{-1}(q)\left[-Y\left(q, \dot{q}, \ddot{q}_{d}\right) \tilde{\theta}\right. \\
\left.+\tilde{M}(q) K \zeta+J^{+}\left(q, \xi_{d}, Z\right) K_{i} \tilde{\xi}+\tau_{N}\right]
\end{aligned}
$$

where $\tilde{\theta}=\theta_{o}-\theta, \tilde{M}(q)=M(q)-M_{o}(q)$ and gain matrix $K$ is chosen such that $(A-B K)$ is Hurwitz. We make the following assumptions to select an additional nonlinear input term and to prove the stability of the close-loop system.

Assumption 1: The norms of the following matrices can be bounded such that

$$
\begin{gathered}
\lambda_{m} \leq\left\|M^{-1}(q)\right\| \leq \lambda_{M}, \lambda_{j} \leq\|J(q, \xi, Z)\| \leq \lambda_{J}, \lambda_{j^{+}} \leq\left\|J^{+}\left(q, \xi_{d}, Z\right)\right\| \leq \lambda_{J^{+}}, \\
K_{m} \leq\|K\| \leq K_{M}, K_{0 m} \leq\left\|K_{0}\right\| \leq K_{0 M}, K_{c m} \leq\left\|K_{c}\right\| \leq K_{c M}
\end{gathered}
$$

Assumption 2: There exist positive constants $\alpha_{M}, \alpha_{C}$ and $\alpha_{G}$ such that

$$
\begin{aligned}
& \left\|M(q)-M_{o}(q)\right\| \leq \alpha_{M} \\
& \left\|C(q, \dot{q})-C_{o}(q, \dot{q})\right\| \leq \alpha_{C}\|\dot{q}\| \\
& \left\|G(q)-G_{o}(q)\right\| \leq \alpha_{G}
\end{aligned}
$$

Assumption 3: There exist nonnegative constants $\beta_{1}, \beta_{2}$ and $\beta_{3}$ such that

$$
\left\|Y\left(q, \dot{q}, \ddot{q}_{d}\right) \tilde{\theta}\right\| \leq \beta_{1}+\beta_{2}\|\zeta\|+\beta_{3}\|\zeta\|^{2}
$$

where $\beta_{1}=\alpha_{M}\left\|\ddot{q}_{d}\right\|+\alpha_{C}\left\|\dot{q}_{d}\right\|^{2}+\alpha_{G}, \beta_{2}=2 \alpha_{C}\left\|\dot{q}_{d}\right\|, \beta_{3}=\alpha_{C}$. 
These assumptions are hold since it is assumed that the desired $q_{d}, \dot{q}_{d}$ and $\ddot{q}_{d}$ belong to the compact set and parametric uncertainties of the robot manipulators are bounded.

We choose the nonlinear control input term $\tau_{N}$ as

$$
\tau_{N}=\left\{\begin{array}{lll}
-\frac{\lambda_{M} \bar{\beta}_{1}(\zeta, \tilde{\xi}) s}{\lambda_{m}\|s\|}-\frac{\bar{\beta}_{2}(\zeta, \widetilde{\xi}) s}{2 \lambda_{m}\|s\|^{2}} & \text { if } & \lambda_{M} \bar{\beta}_{1}(\zeta, \widetilde{\xi})\|s\| \geq \mu \\
-\frac{\lambda_{M}^{2} \bar{\beta}_{1}^{2}(\zeta, \widetilde{\xi}) s}{\lambda_{m} \mu}-\frac{\bar{\beta}_{2}(\zeta, \tilde{\xi}) s}{2 \lambda_{m}\|s\|^{2}} & \text { if } & \lambda_{M} \bar{\beta}_{1}(\zeta, \widetilde{\xi})\|s\|<\mu
\end{array}\right.
$$

where $\mu>0$ is a design parameter to be chosen, $s=B^{T} P \zeta$,

$$
\begin{aligned}
& \bar{\beta}_{1}(\zeta, \tilde{\xi})=\beta_{1}+\beta_{2}\|\zeta\|+\beta_{3}\|\zeta\|^{2}+\alpha_{M} K_{M}+\lambda_{J^{+}} K_{M}\|\tilde{\xi}\|, \text { and } \\
& \bar{\beta}_{2}(\zeta, \widetilde{\xi})=\lambda_{J} K_{o M}\|\widetilde{\xi}\|\|\zeta\| .
\end{aligned}
$$

Theorem 1: Suppose that assumption 1, 2 and 3 hold. Given the error dynamic equations (5) and (15), the proposed controller (13) with (21) ensures position tracking errors to be globally uniformly bounded.

Proof: Consider the Lyapunov function candidate

$$
V=\zeta^{T} P \zeta+\frac{1}{2} \widetilde{\xi}^{T} K_{0} \xi
$$

where $K_{0}$ is the positive definite matrix and $P=P^{T}>0$ is the solution of the Lyapu nov equation $(A-B K)^{T} P+P(A-B K)=-I$

Define the set $\Omega_{c}$ include the initial value

$$
\Omega_{c}=\left\{(\zeta, \tilde{\xi}) \in R^{3 n+2} \mid V(\zeta, \tilde{\xi}) \leq c\right\}, c>0
$$

The time derivative of $V$ along the trajectories of the equations (12) and (15) yields

$$
\begin{aligned}
& \dot{V}= \dot{\zeta}^{T} P \zeta+\zeta^{T} P \dot{\zeta}+\widetilde{\xi}^{T} K_{0} \dot{\vec{\xi}} \\
& \leq-\|\zeta\|^{2}-K_{o m} \lambda_{j} \lambda_{j}{ }_{j} K_{c m}\|\widetilde{\xi}\|^{2}+2 \lambda_{M}\|s\| \bar{\beta}_{1}(\cdot) \\
& \\
&+\bar{\beta}_{2}(\cdot)+2 s^{T} M^{-1}(q) \tau_{N}
\end{aligned}
$$

If $\lambda_{M} \bar{\beta}_{1}(\zeta, \tilde{\xi})\|s\| \geq \mu$, we have

$$
\dot{V} \leq-\|\zeta\|^{2}-\eta\|\widetilde{\xi}\|^{2}
$$

where $\eta=K_{o m} \lambda_{j} \lambda_{j^{+}} K_{c m}$. 
If $\lambda_{M} \bar{\beta}_{1}(\zeta, \widetilde{\xi})\|s\|<\mu$, we have

$$
\dot{V} \leq-\|\zeta\|^{2}-\eta\|\tilde{\xi}\|^{2}+\frac{\mu}{2}
$$

For $\mu \leq \frac{4 \eta c}{a \lambda_{\min }(P) K_{o m}}$ with $a>1$

$$
\dot{V} \leq 0
$$

If we define the set $\Omega_{\mu}=\left\{\lambda_{M} \bar{\beta}_{1}(\zeta, \tilde{\xi})\|s\|<\mu\right\}$, from (27) the trajectory $(\zeta, \tilde{\xi})$ will enter the set $\Omega_{\mu}$ in infinite time and remain thereafter.

Therefore, the control law (13) with (21) guarantees the uniformly ultimate boundedness of the closed-loop system.

\section{Simulation and Experimental Results}

The proposed control method was implemented on 2-link robot manipulator manufactured by Samsung Faraman-AS1. The dynamic equation of the manipulators is given by

$$
\left[\begin{array}{ll}
M_{11}(q) & M_{12}(q) \\
M_{12}(q) & M_{22}(q)
\end{array}\right]\left[\begin{array}{l}
\ddot{q}_{1} \\
\ddot{q}_{2}
\end{array}\right]+\left[\begin{array}{cc}
0 & C_{12}(q, \dot{q}) \\
C_{21}(q, \dot{q}) & 0
\end{array}\right]\left[\begin{array}{l}
\dot{q}_{1} \\
\dot{q}_{2}
\end{array}\right]+\left[\begin{array}{c}
G_{1}(q) \\
G_{2}(q)
\end{array}\right]=\left[\begin{array}{c}
\tau_{1} \\
\tau_{2}
\end{array}\right]
$$

Setup for experiments is shown in Fig. 1. A motion control board(MMC) mounted in a main computer is used to execute the control algorithm. The feature signal is acquired by a image processing board(Matrox Meteor II) mounted on a main computer which processes the image obtained from a CCD camera and extracts the image feature. The CCD camera is mounted on the end-effector. The image obtained by the image processor has a $640 \times 480$ pixels resolution

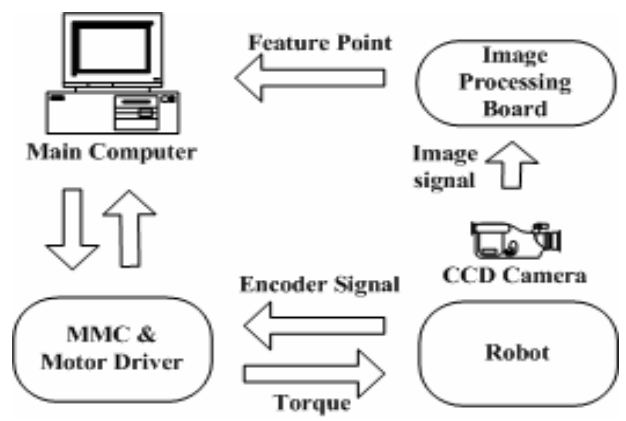

Fig. 1. Block diagram of vision systems 
The variation interval of the parameter due to an unknown load is considered

$$
0 \leq \Delta m_{4} \leq 4.5 \mathrm{~kg}, 0 \leq \Delta I_{2} \leq 0.005 \mathrm{Kgm}^{2}
$$

In the experimental test, we have considered one object feature point on a whiteboard. The white board was located at a distance $Z=1 \mathrm{~m}$ in front of the camera and parallel to the plane where the manipulator moves. The controller gain matrices $K_{c}$ and $K$ are chosen as $K_{c}=10 I$ and $K=[2 I 50 I 50 I]$, respectively. The control gains $K_{i}$ and $K_{o}$ are chosen as $K_{i}=7 \times 10^{-8}$ and $K_{o}=7 \times 10^{-8}$, respectively. The initial positions of each joint are $q_{1}=\pi / 2(\mathrm{rad}), q_{2}=\pi(\mathrm{rad})$. It was assumed that the initial feature point of the object was $\xi=[100100]^{T}$ pixels. The desired feature point coordinate was $\xi_{d}=\left[\begin{array}{ll}0 & 0\end{array}\right]^{T}$ pixels.

The simulation results are shown in Figs. 2, 3 and 4. Fig. 2 illustrates the trajectory of feature point on the image plane which shows the convergence to the desired feature

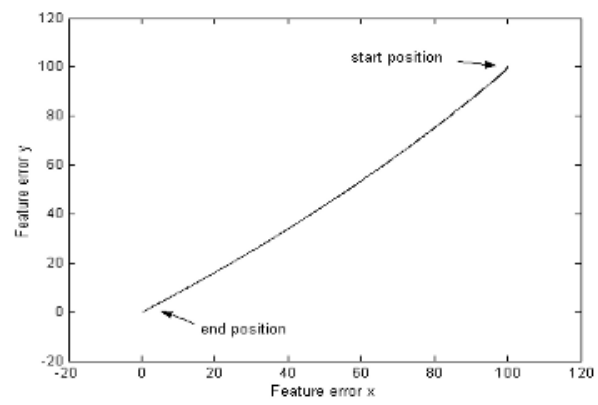

Fig. 2. Simulation results: Trajectories of feature errors using integral action(solid) and without integral action(dashed)

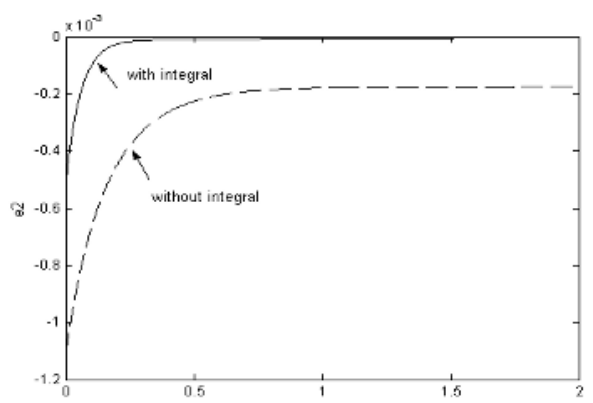

Fig. 4. Simulation results: Tracking errors of link 2 using integral action(solid) and without $\mathrm{i}$ ntegral action(dashed)

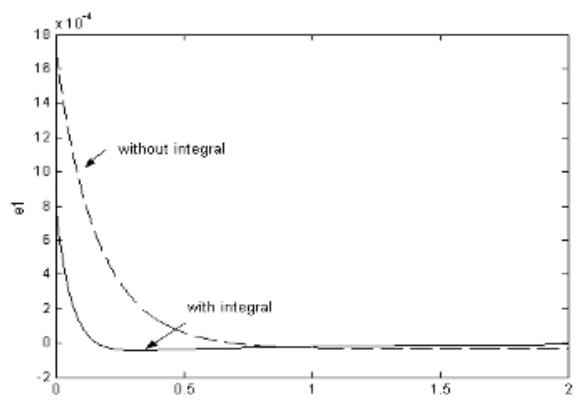

Fig. 3. Simulation results: Tracking errors of link 1 using integral action(solid) and without integral action(dashed)

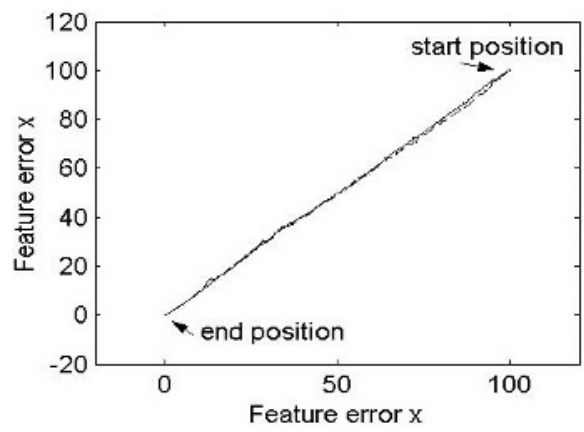

Fig. 5. Experimental results: Trajectories of feature errors using integral action(solid) and without integral action(dashed) 
point. Fig. 3, 4 represents the tracking errors of joint 1 and 2. The results by the proposed method are compared to those without integral action. It is shown that better tracking performance results are archived by the robust controller with integral actions.

Figs. 5, 6 and 7 are experimental results that the proposed control algorithm applies to the 5-link Samsung Faraman robot. Experimental results show that the tracking performance is effective.

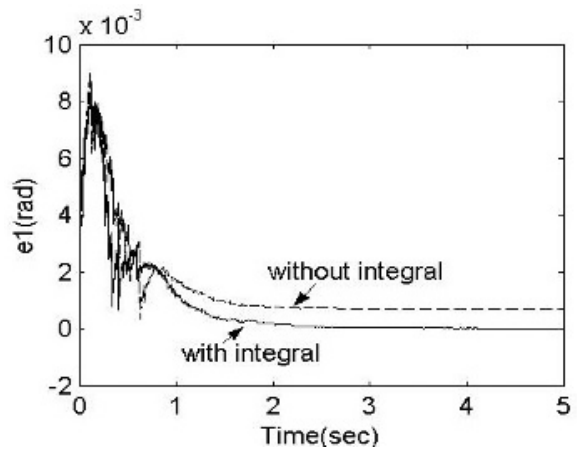

Fig. 6. Experimental results: Tracking errors of link 1 using integral action(solid) and without integral action(dashed)

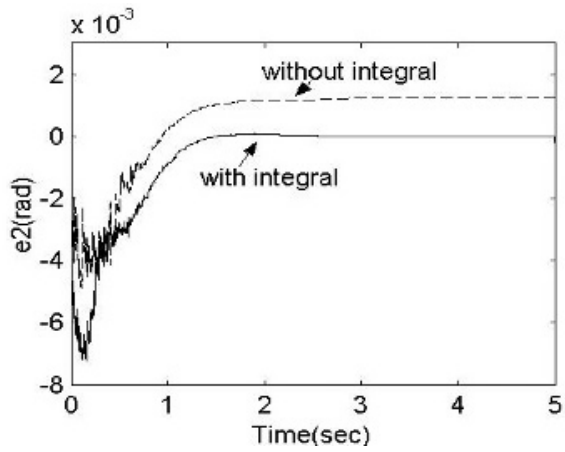

Fig. 7. Experimental results: Tracking errors of link 2 using integral action(solid) and without integral action(dashed)

\section{Conclusions}

In this paper, a robust controller with visual feedback for robot manipulators was proposed. The controller is of structure based on the image feature errors and the joint velocities fed back by the CCD camera and the encoder, respectively. The proposed controller with integral action reduces tracking error due to parametric uncertainties.

The ultimate uniform stability of the overall closed-loop system is proved by using the Lyapunov method. Simulation and Experiment results on a two degree of freedom manipulator have shown that the proposed control method has effectiveness to control robot manipulators with uncertainty.

\section{References}

1. Hashimoto, K.: VISUAL SERVOING. World Scientific (1993)

2. Espiau, E., Chaumette, F., Rives, P.: A new approach to visual servoing in robotics. IEEE Trans. Robotics and Automation, Vol. 8, No.3 (1992) 313-326

3. Hashimoto, K., Kimoto, T., Ebine, T., Kimura, H.: Manipulator control with image-based visual servo. IEEE International Conference on Robotics and Automation (1991) 2267-2272 
4. Kelly, R., Carelli, R., Nasisi, O., Kuchen, B., Reyes, F.: Stable visual servoing of camerain-hand robotic systems. IEEE/ASME Trans. Mechatronics, Vol. 5, No.1, (2000) 39-43

5. Zergeroglu, E., Dawson, D. M., Queiroz, M. S. de., Setlur, P.: Robust visual-servo control of robot manipulators in the presence of uncertainty. Journal of Robotic Systems, Vol. 20, issue, 2(2003) 93-106

6. Liu, G. J.,Goldenberg, A. A., Robust control of robot manipulators based on dynamic decomposition. IEEE Trans. Robotics and Automation, vol. 13, no. 5, (1997) 783-789

7. Spong, M. W., Vidyasagar, M.: Robot Dynamics and Control. Wiley, NewYork (1989) 